\title{
Arquitectura de un Dispositivo no Invasivo para el Seguimiento de la Conservación de Alimentos en Empaques Inteligentes.
}

\section{Architecture of a Device Non-Invasive for the Monitoring of Food Conservation in the Intelligent Packaging.}

\author{
Jairo Ramón Valencia¹.; Cecilia Murrugarra².; Cristian Villa Zabala³.;Martha P. \\ Tarazona-Díaz ${ }^{4}$ \\ ${ }^{1}$ Universidad El Bosque. Grupo de investigación OSIRIS \& BIOAXIS, leninramon@unbosque.edu.co \\ 2 Universidad El Bosque. Grupo de investigación Electromagnetismo, Salud y Calidad de Vida, \\ cmurrugarra@unbosque.edu.co \\ 3 Universidad del Quindío. Grupo de investigación Ciencia y Tecnología de Alimentos CYTA, \\ ccvilla@uniquindio.edu.co \\ 4 Universidad Jorge Tadeo Lozano. Grupo de investigación en Procesos y sistemas industriales, \\ marthap.tarazonad@utadeo.edu.co
}

\section{Resumen}

Este estudio está centrado en plantear una arquitectura del hardware de un dispositivo electrónico que sirve para el diagnóstico de la conservación de las carnes envasadas con empaques inteligentes, el grupo de trabajo está conformado por un equipo multidisciplinario que es capaz de realizar la extracción y caracterización de los antioxidantes de la mora, fabricar y caracterizar el gel a partir de almidón o alginato de mora, que es usado en el empaque de alimento de modo que se puede realizar el reconocimiento de los cambios de color del gel y conocer la variación de la composición del gel al estar en contacto con el alimento. Para esta fase fue necesario desarrollar un dispositivo electrónico no invasivo, capaz de leer los cambios del color del gel. Como resultado preliminar se realizaron pruebas de calibración y caracterización de la arquitectura propuesta basada en el sensor conversor de luz a frecuencia TCS 3210/3200. Con esta arquitectura se identificaron los cambios de coloración del gel en un empaque inteligente, para finalmente parametrizar otras variables ambientales que es susceptible a cambios en la variación del $\mathrm{pH}$ del empaque inteligente.

Palabras clave: antocianina, antioxidantes, biosensor, indicador $\mathrm{pH}$.

\begin{abstract}
In this study, the focus is on designing an architecture of an electronic device that serves to diagnose the conservation of packaged meats with intelligent packaging, the working group is made up of a multidisciplinary team that is able to perform the extraction and characterization of the blackberry antioxidants, manufacture and characterize the gel from blackberry starch or alginate, which is used in the food packaging so that the recognition of gel color changes can be realized and the variation of the composition of the gel can be known gel when in contact with the food. For this phase it was necessary to develop a non-invasive electronic device, capable of reading the gel color changes. As a preliminary result, calibration and characterization tests of the proposed architecture were carried out based on the TCS 3210/3200 frequency converter light sensor. This architecture will identify the changes in the color of the gel in an intelligent packaging, in order to finally parameterize other environmental variables that are susceptible to changes in the variation of the $\mathrm{pH}$ film.
\end{abstract}

Keywords: anthocyanins, antioxidants, biosensor, $\mathrm{pH}$ indicator 


\section{Introducción}

La industria de los alimentos ha venido desarrollando una serie de empaques para alimentos, cuyo principal fin, es extender el tiempo de vida de los alimentos. Existe una gran variedad de biomateriales que son utilizados para la fabricación de las películas o recubrimientos de los empaques inteligentes. El empaque inteligente suministra información directa de la calidad del producto y tienen la capacidad de detectar, medir, grabar, generar trazas de parametrización de variables químicas, comunicación de datos (transferencia de la información) o una aplicación científica determinada. Estas características de los empaques inteligentes permiten extender el tiempo de permanencia de los alimentos en las góndolas de comercialización, mejorar la seguridad de consumo de los alimentos, monitorear la calidad del alimento y alertar sobre posibles problemas o cambios de los parámetros para el consumo humano (Brody, Strupinsky, \& Kline, 2001; Fuertes et al., 2016) (Arvanitoyannis \& Oikonomou, 2012; Lavoine, Desloges, Manship, \& Bras, 2015), (Biji, Ravishankar, Mohan, \& Srinivasa Gopal, 2015; Brody et al., 2001). Dentro de los empaques inteligentes se encuentran biosensores habilitados para detectar variables químicas capaces de mostrar el grado de degradación del alimento y en algunos casos variables sensibles a la visualización de los cambios de los indicadores medibles en el tiempotemperatura, éstos logran medir la integridad del alimento con la activación de una bio-película de gel que reacciona ante el cambio de $\mathrm{pH}$ del alimento con la etiqueta que contiene el gel. García \& Pinzón, (2016) realizaron recubrimientos a base de almidón de plátano Guayabo y otros aditivos, aplicados sobre frutos frescos de fresa y evaluaron el efecto de estos recubrimientos en el comportamiento postcosecha de las fresas durante el almacenamiento en condiciones de refrigeración. El biosensor reacciona ante los cambios que se producen en el alimento como resultado del crecimiento de microorganismos.

Existen diferentes técnicas usadas en la industria alimentaria para determinar las propiedades fisicoquímicas de los alimentos, dentro de ellas se encuentra la espectroscopia, sensores de identificación por radiofrecuencia, visión por computadora (Ruiz-Altisent et al., 2010). La visión artificial por computadora es una de las técnicas que se ha venido utilizando en la industria de alimentos, ya que permite una alternativa para una técnica automatizada, no destructiva, no invasiva y rentable para cumplir con estos requisitos (Brosnan \& Sun, 2004). Otras de las técnicas utilizadas en el ámbito de la industria alimentaria es el uso de imágenes hiperespectrales, en este caso Liu et al. (2014), realizaron un método rápido, preciso y confiable para el control y calidad de las etapas de maduración de la carne. Para ello, se usaron técnicas de imagen hiper-espectrales en longitudes de onda entre $400 \mathrm{~nm}-1000 \mathrm{~nm}$, para predecir el color y $\mathrm{pH}$ de la carne, a partir de modelos estadísticos que permitieran relacionar ciertos subconjuntos de ondas que contenían información relevante para el desarrollo del modelo (Elmasry, Sun, \& Allen, 2012). El uso de ondas por ultrasonido ha tenido en los últimos años diversas aplicaciones en el ámbito industrial, gracias a que esta técnica resulta bastante fácil de implementar, es económica y permite un ahorro en cuanto a energía se refiere, es por ello que se planteó una aplicación para la industria alimentaria que permitiera monitorear la composición y propiedades químicas de los alimentos durante su procesamiento $\mathrm{y}$ almacenamiento (Awad, Moharram, Shaltout, Asker, \& Youssef, 2012). Por último, dentro de las técnicas que se han usado en la industria alimentaria, se encuentran las ondas electromagnéticas que desde su amplia gama, permite una multitud de posibles desarrollos que permiten lograr un seguimiento de las propiedades fisicoquímicas de los alimentos, todo esto con el fin de preservar su calidad y conservar lo más posible su frescura con la intención de 
ofrecer seguridad al momento del consumo (Damez \& Clerjon, 2013).

Este estudio se basa en plantear una arquitectura del hardware de un dispositivo electrónico, portable, no invasivo y de bajo costo que pueda ser utilizado en mejorar la calidad y conservación de alimentos envasados en los empaques inteligentes. Para ello se realizará la extracción y caracterización de los antioxidantes de la mora, se desarrollará y caracterizará el gel a partir del almidón o alginato, que es usado en la elaboración del empaque que recubre el alimento, de modo que, cuando el alimento cárnico se encuentra en contacto con el empaque, este reacciona cambiando su coloración de acuerdo al pH del alimento.

El dispositivo electrónico realizará el reconocimiento de los cambios de color del gel y permitirá identificar la variación de la composición del gel al estar en contacto con el alimento. Como resultado preliminar se realizaron pruebas de calibración y caracterización de la arquitectura propuesta basada en el sensor conversor de luz a frecuencia TCS 3210/3200 (Ams, 2016), (Bishop \& Lee, 2006). Con esta arquitectura se identificará los cambios de coloración del gel en un empaque inteligente, para finalmente parametrizar otras variables ambientales que sean susceptibles a los cambios de la variación de la película del $\mathrm{pH}$.

\section{Metodología}

Para el diseño e implementación del dispositivo no invasivo en el seguimiento de la conservación de alimentos en empaques inteligentes se establecieron tres etapas 1) Extracción de antiocianinas. 2) Hidrogel para empaques inteligentes y 3) Arquitectura del dispositivo no Invasivo. A continuación, se detalla cada una de las etapas desarrolladas:

\subsection{Extracción de Antocianinas}

Para obtener un extracto en solvente de antocianinas, factores como el $\mathrm{pH}$, el tiempo de extracción, la relación materia prima-solvente y la temperatura son influyentes durante el proceso, ya que afectan la difusividad de la antocianina en el medio y su estabilidad. Por ello, en este estudio se trabajó con corteza de mora de dos variedades; castilla y silvestre. Las cortezas fueron sometidas a extracción de contacto simple con etanol al
96\%, acidificado con ácido cítrico a $\mathrm{pH} 2,8$ durante $142,5 \min$ a $57,5^{\circ} \mathrm{C}$. La extracción de la muestra se realizó de acuerdo al método usado por Fan et al. (2008) ajustado a las condiciones del laboratorio.

En primer lugar, se realizó la medición de temperatura de la mezcla solvente-materia prima, para ello se empleó un termómetro digital con un rango de $\quad-50 \quad{ }^{\circ} \mathrm{C}$ a $150 \quad{ }^{\circ} \mathrm{C}$ (Long-Stem Thermometers Traceable, Georgia, Estados Unidos). Se pesó la materia prima en una balanza de precisión (Explorer pro Ohaus, México) y se midió el $\mathrm{pH}$ de la solución con un $\mathrm{pH}$ metro/ionómetro de precisión de $\mathrm{pH}$ relativo de \pm 0,002 (SevenCompact ${ }^{\mathrm{TM}}$ S220, Switzerland, Suiza). Se empleó como solvente etanol al $96 \%$ (V/V), con pH neutro, el cual se acidificó con ácido cítrico.

Finalmente, se centrifugó la muestra (4000 RPM por $15 \mathrm{~min}$ ) en una centrifuga (Hettich rotofix 32 zentrifugen, Schwerin, Alemania).

\subsection{Hidrogel para empaques inteligentes}

Para la formación del hidrogel se emplearon como matrices poliméricas, almidón de plátano, variedad guayabo y alginato de sodio. Para esto se preparó una solución filmogénica con $3 \%$ de almidón y $2 \%$ de alginato, las cuales fueron calentadas con agitación mecánica hasta $80{ }^{\circ} \mathrm{C}$, posteriormente se agregó el extracto de antocianinas para alcanzar una concentración final del $15 \%$. La mezcla filmogénica fue vertida en un recipiente de acrílico y secada en una estufa con recirculación de aire caliente a $35^{\circ} \mathrm{C}$ durante 24 horas.

\subsection{Arquitectura del dispositivo.}

En la Figura 1, se muestran los 4 módulos que conforman la arquitectura del dispositivo no invasivo que determina la calidad del alimento cárnico. En las siguientes subsecciones se describe cada uno de los módulos del dispositivo desarrollado:

\subsubsection{Módulo Sistema de Adquisición.}

El módulo sistema de adquisición es el encargado de recibir la información del alimentocubierto por el empaque inteligente (hidrogel a base del 
alginato de mora). Para iniciar el proceso de reconocimiento del alimento, el módulo recibe una serie de señales de control y habilitación para que a partir del arreglo de $8 \times 8$ fotodiodos del Sensor TCS3200(Ams, 2016), se realice una conversión de luz a frecuencia, de modo

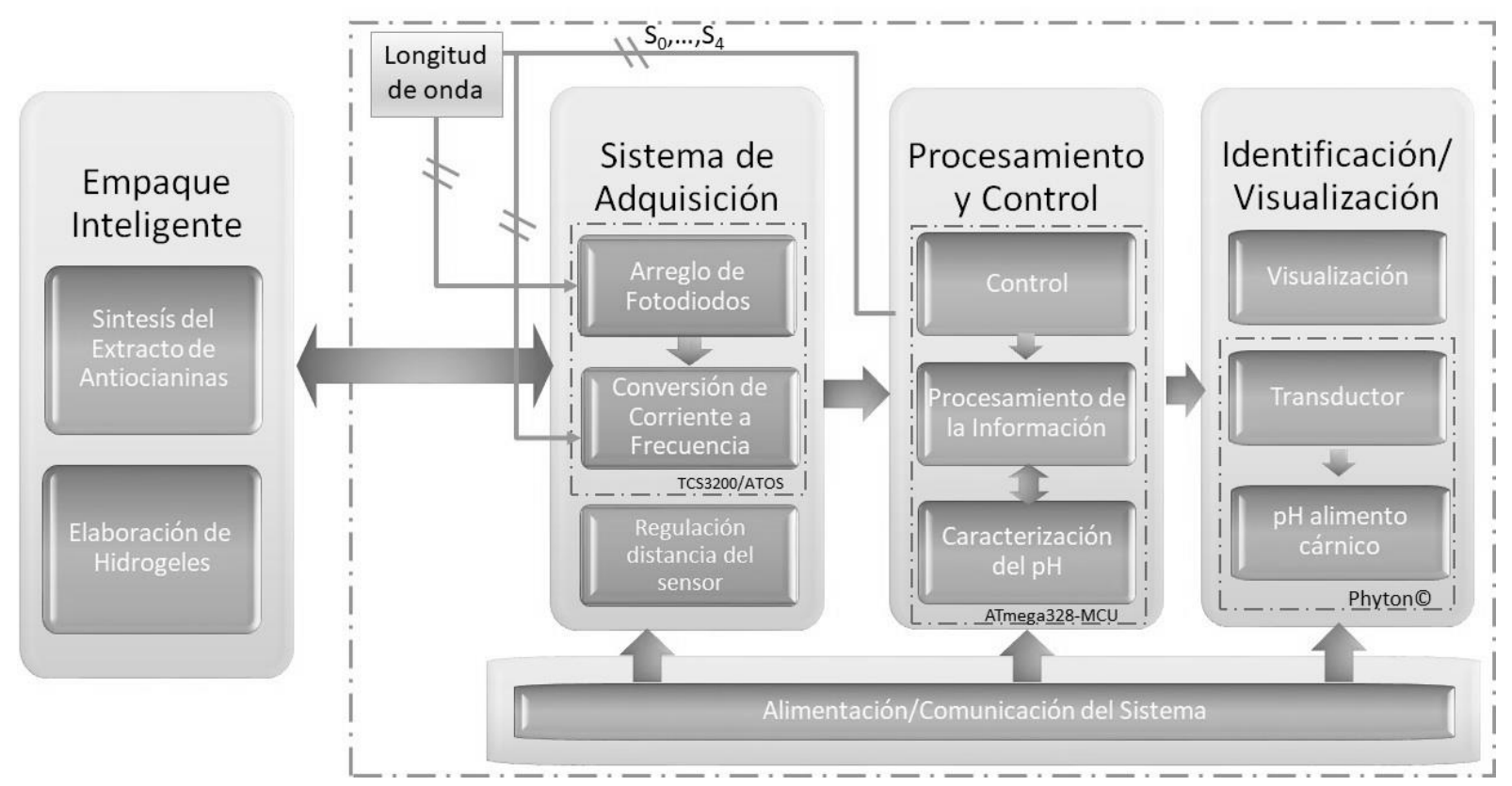

Figura 1. Diagrama General de la Arquitectura de un Dispositivo no Invasivo para el Seguimiento de la Conservación de Alimentos en Empaques Inteligentes.

que se activarán tres etapas de filtrado para determinar las componentes RGB (Rojo, Verde, y Azul) del color del alimento. El módulo de adquisición también cuenta con un mecanismo para calibrar la distancia vertical entre el alimento y el sensor TC3200, y de esta manera garantizar las condiciones de toma de información de cada empaque de alimento.

\subsubsection{Módulo Procesamiento y Control.}

El módulo de procesamiento y control es el encargado de activar la secuencia de líneas para establecer comunicación con el Sensor TCS3200, determina la secuencia de instrucciones para realizar el filtrado de señales para obtener las componentes RGB. Este módulo tiene como núcleo para su procesamiento un microcontrolador basado en el procesador ATmega328 MCU. Del procesador se emplean cuatro salidas digitales $S_{0}, S_{1}, S_{2}$ y $S_{3}$, las dos primeras señales son usadas para definir la escala de frecuencia de salida $f_{0}$, y las dos últimas señales, permiten seleccionar el arreglo de fotodiodos de acuerdo al color RGB a filtrar para determinar el color en el módulo de adquisición del sistema. El módulo de Procesamiento y Control tiene una señal de entrada digital que le permite al núcleo de procesamiento obtener la respuesta del Sistema de Adquisición y poder comenzar el proceso de Caracterización del $\mathrm{pH}$ del alimento en función de las características de acidez o basicidad del alimento escaneado, en este caso el alimento cárnico (Fellows, 2009).

\subsubsection{Módulo Identificación/Visualización}

Una vez realizado el proceso de filtraje para cada una de las componentes RGB, éste será utilizado para caracterizar los diferentes tipos de $\mathrm{pH}$ del alimento y se comienza el proceso de identificación de acuerdo a las tablas de 
caracterización del hidrogel desarrollado y con el soporte UltraBasic Benchtop $\mathrm{pH}$ Meter (Instruments, 2013), y los valores identificados por el módulo del sistema de adquisición, para proceder a indicar en una pantalla al usuario las características del alimento escaneado.

\subsubsection{Módulo de Alimentación / Comunicación}

El dispositivo cuenta con un banco de baterías tipo Litio y Polímero (LiPo), para una autonomía de 8 horas. El módulo de comunicación del dispositivo utiliza el módulo Bluetooth HC-05 con protocolo UART RS 232 serial.

\section{Resultados y Discusión.}

\subsection{Resultados preliminares extracción de antocianinas}

La variedad de mora silvestre presentó mayor contenido de antocianinas $(28.58 \mathrm{mg} / 100 \mathrm{~g})$ en comparación con la mora variedad castilla (Tabla 1). La mora silvestre al ser considerada un desecho (maleza) podría ser rentable en su utilización como materia prima para la extracción de antocianinas empleadas como indicador de $\mathrm{pH}$ en empaques inteligentes.

Tabla1. Resultados preliminares extracción de antocianinas.

\begin{tabular}{|c|c|}
\hline Variedad & Antocianinas mg/100 g \\
\hline Mora Castilla & $16.75 \pm 2.34$ \\
\hline Mora Silvestre & $28.58 \pm 2.23$ \\
\hline
\end{tabular}

Promedio \pm D.E $(n=3)$

\subsection{Resultados preliminares dispositivo no invasivo e hidrogel.}

Se realizaron pruebas preliminares con el hidrogel activado con varias soluciones de Hidróxido de Sodio $(\mathrm{NaOH})$ en varias concentraciones con el objetivo modificar el $\mathrm{pH}$ de las muestras del hidrogel colocados en placas de Petri, una vez calculadas las concentraciones de $\mathrm{NaOH}$ para el rango de $\mathrm{pH}$ entre [5,5.6], haciendo uso de un $\mathrm{pH}$ Meter UB-10 de Denver Instrument, se practicaron pruebas de medición con el dispositivo no invasivo, obteniéndose resultados de las componentes RGB para cada uno de los valores de pH, como se muestra en la Figura 12.

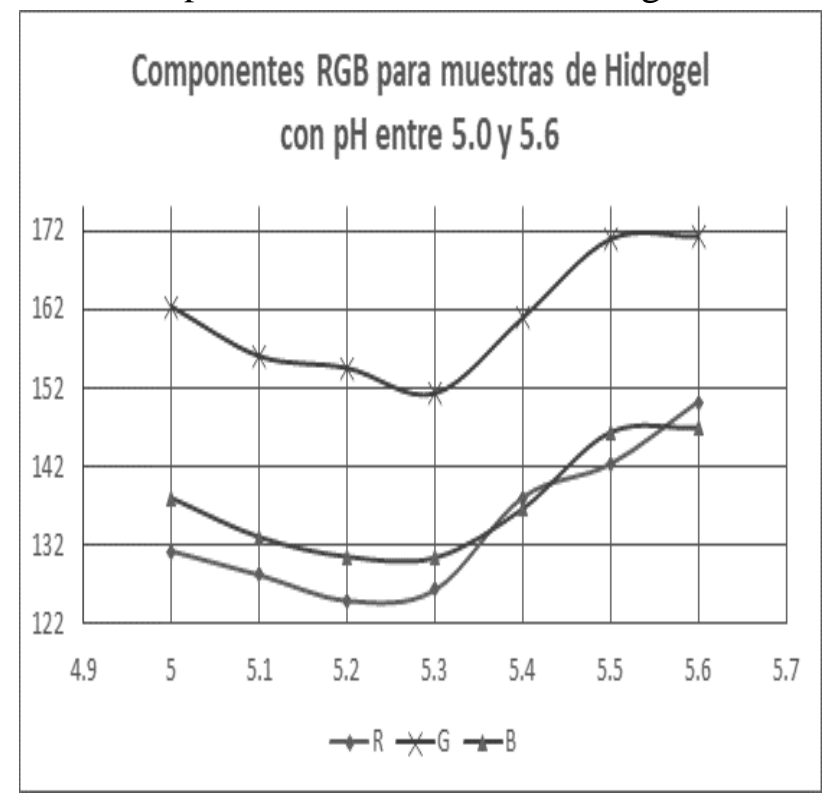

Figura 2. Componentes $R G B$ de pruebas preliminares realizadas con el Dispositivo no Invasivo

Como se muestra en la Figura 2, el dispositivo logra detectar diferentes niveles de las componentes RGB para cada uno de los hidrogeles reaccionados para diferentes concentraciones de $\mathrm{NaOH}$.

\section{Conclusiones}

El extracto de antocianina de mora tanto de variedad castilla $(16.75 \mathrm{mg} / 100 \mathrm{~g})$ como silvestre $(28.58 \mathrm{mg} / 100 \mathrm{~g})$ puede ser utilizado como indicador visual de cambios $\mathrm{pH}$, para comunicar el deterioro de productos alimenticios debido a sus reacciones fisicoquímicas y fisiológicas.

\section{Agradecimientos}

A los investigadores pertenecientes a la facultad de ingeniería Universidad El Bosque, en especial a los programas de Ingeniería Electrónica y Bioingeniería, por la ejecución del estudio y el desarrollo del proyecto interdisciplinario. Además, agradecemos al personal técnico del "Laboratorio de Química", Facultad de Ciencias de la Universidad El Bosque, por permitir el uso 
quality attributes using electromagnetic

de sus instalaciones para realizar pruebas y mediciones con el prototipo en desarrollo.

\section{Referencias Bibliogràfícas}

Ams, S. (2016). TCS3200 Color Sensor: Programmable Color Light-to-Frequency Converter. AMS. Retrieved from http://www.austriamicrosystems.org/eng/Pr oducts/Light-Sensors/Color-

Sensors/TCS3200/TCS3200-TCS3210-

Datasheet

Arvanitoyannis, I., \& Oikonomou, G. (2012). Active and Intelligent Packing and its New Trends. In I. Arvanitoyannis (Ed.), Modified Atmosphere and Active Packing Technologies (pp. 628-654). Florida: CRC Pres.

Awad, T. S. S., Moharram, H. A. A., Shaltout, O. E. E., Asker, D., \& Youssef, M. M. M. (2012). Applications of ultrasound in analysis, processing and quality control of food: A review. Food Research International.

https://doi.org/10.1016/j.foodres.2012.05.0 04

Biji, K. B., Ravishankar, C. N., Mohan, C. O., \& Srinivasa Gopal, T. K. (2015). Smart packaging systems for food applications: a review. Journal of Food Science and Technology. https://doi.org/10.1007/s13197-015-1766-7

Bishop, T., \& Lee, G. (2006). White Paper: TAOS Colorimetry Tutorial. AMS. Retrieved from http://www.austriamicrosystems.org/eng/co ntent/view/download/145161

Brody, A. L., Strupinsky, E. R., \& Kline, L. R. (2001). Active Packaging for Food Applications. Active Packaging for Food Applications.

https://doi.org/10.1017/CBO978110741532 4.004

Brosnan, T., \& Sun, D. W. (2004). Improving quality inspection of food products by computer vision - A review. Journal of Food Engineering.

https://doi.org/10.1016/S0260-

8774(03)00183-3

Damez, J.-L., \& Clerjon, S. (2013). Quantifying and predicting meat and meat products waves: an overview. Meat Science. https://doi.org/10.1016/j.meatsci.2013.04.0 37

Elmasry, G., Sun, D. W., \& Allen, P. (2012). Near-infrared hyperspectral imaging for predicting colour, $\mathrm{pH}$ and tenderness of fresh beef. Journal of Food Engineering. https://doi.org/10.1016/j.jfoodeng.2011.11. 028

Fan, G., Han, Y., Gu, Z., \& Chen, D. (2008). Optimizing conditions for anthocyanins extraction from purple sweet potato using response surface methodology (RSM). LWT - Food Science and Technology, 41(1), 155-160. https://doi.org/10.1016/j.lwt.2007.01.019

Fellows, P. J. (2009). Food Processing Technology. Food Processing Technology. https://doi.org/10.1533/9781845696344.2.2 90

Fuertes, G., Soto, I., Carrasco, R., Vargas, M., Sabattin, J., \& Lagos, C. (2016). Intelligent Packaging Systems: Sensors and Nanosensors to Monitor Food Quality and Safety. Journal of Sensors. https://doi.org/10.1155/2016/4046061

García, O., \& Pinzón, M. (2016). EFECTO DE RECUBRIMIENTOS DE ALMIDÓN DE PLÁTANO GUAYABO (Musa paradisiaca L.) EN LA CALIDAD DE FRESAS. Revista Alimentos Hoy, 24(30), 92-102. https://doi.org/http://alimentoshoy.acta.org. co/index.php/hoy/article/view/407

Instruments, D. (2013). UltraBasic Benchtop pH Meter. Retrieved from http://www.denverinstrument.com/denveru sa/media/pdf/OpManUBLongRevA.pdf

Lavoine, N., Desloges, I., Manship, B., \& Bras, J. (2015). Antibacterial paperboard packaging using microfibrillated cellulose. Journal of Food Science and Technology. https://doi.org/10.1007/s13197-014-1675-1

Liu, D., Ma, J., Sun, D. W., Pu, H., Gao, W., Qu, J., \& Zeng, X. A. (2014). Prediction of Color and $\mathrm{pH}$ of Salted Porcine Meats Using Visible and Near-Infrared Hyperspectral Imaging. Food and Bioprocess Technology. https://doi.org/10.1007/s11947-014-1327-5

Ruiz-Altisent, M., Ruiz-Garcia, L., Moreda, G. P., Lu, R., Hernandez-Sanchez, N., Correa, 
E. C., ... García-Ramos, J. (2010). Sensors for product characterization and quality of specialty crops-A review. Computers and Electronics in Agriculture.

https://doi.org/10.1016/j.compag.2010.07.002

*Para citar este artículo: Ramón Valencia R.; Murrugarra C.; Villa Zabala C.;Tarazona-Díaz M.P . Architecture of a Device Non-Invasive for the Monitoring of Food Conservation in the Intelligent Packaging. Revista Bistua.2018.16(1):103-109.

+ Autor para el envió de correspondencia y la solicitud de las separatas: Ramón Valencia R, Universidad dad El Bosque. Grupo de investigación OSIRIS \& BIOAXIS, leninramon@unbosque.edu.co

Recibido: Enero 18 de 2017

Aceptado:Enero 30 de 2018 